\title{
Una mirada al fenómeno de la violencia escolar en Costa Rica
}

\author{
http://doi.org/10.15359/ree.13-1.4 \\ Leonel Arias Sandoval \\ Docente en la División de Educología de la Universidad Nacional de Costa Rica \\ Heredia, Costa Rica
}

Recibido 30 de julio de 2008 • Aprobado 23 de marzo de 2009

\begin{abstract}
Resumen. En el actual contexto educativo, padres de familia, estudiantes, docentes, medios de comunicación y demás sectores de la sociedad costarricense, manifiestan su preocupación ante el problema de la violencia escolar, fenómeno social que ha venido en aumento en los últimos años. Sus manifestaciones se perciben por medio de conductas de maltrato, intimidación, agresión verbal o física entre jóvenes, y que se construye por medio de prácticas culturales. De ahí que, para comprender este problema, se debe reflexionar acerca de las posibles causas, teniendo en cuenta el contexto en el cual se desarrolla la convivencia social en cada centro educativo. Algunas de las manifestaciones de violencia tienen su origen en la familia, en la comunidad, en la imitación de patrones de conducta y en la influencia de los medios de información de masa. Además, estos comportamientos son potenciados por el modelo curricular vigente, lo que genera resistencia a las normas institucionales.
\end{abstract}

Palabras clave. Violencia escolar, cultura escolar, interacciones, currículo oculto, educación, medios de comunicación.

Abstract. In the current context of education, parents, students, teachers, media and other sectors of Costa Rican society, express concern about the problem of school violence, a social phenomenon that has been increasing in recent years. Its manifestations are perceived by means of abuse behavior, intimidation, verbal or physical abuse among youth, which are constructed through cultural practices. Therefore, to understand this problem, reflection about possible causes is in order, taking into account the context in which social interaction is developed in each school. Some of the manifestations of violence are rooted in the family, the community, the imitation of the behavioral patterns, and the influence of mass media. Moreover, these behaviors are reinforced by the current curriculum model, generating resistance to institutional rules.

Key words. School violence, school culture, interactions, hidden curriculum, education, media.

Magíster en Psicopedagogía por la Universidad Estatal a Distancia. Licenciado en Ciencias de la Educación con Énfasis en Didáctica por la Universidad Nacional de Costa Rica. Bachiller en la Enseñanza de los Estudios Sociales por la Universidad Nacional de Costa Rica. Actualmente, se desempeña como docente en la División de Educología de la Universidad Nacional de Costa Rica y como Coordinador Académico del Liceo el Carmen de Alajuela. Correo electrónico: ariasleonel@yahoo.com 


\section{CONSIDERACIONES PRELIMINARES}

En el contexto educativo, padres de familia, estudiantes, profesores, medios de comunicación y demás sectores de la sociedad nacional, expresan su preocupación por el problema de la violencia escolar, fenómeno social que ha venido aumentando durante los últimos años. De ahí que, para comprender este problema, se debe reflexionar acerca de las posibles causas, teniendo en cuenta el contexto sociocultural en el cual se desarrolla la cotidianidad de cada centro educativo y las relaciones sociales que en ella se den.

Las manifestaciones de la violencia escolar repercuten fuertemente en el país y produce alarma social, por cuanto son llamativas a la prensa sensacionalista; cada vez es mayor la frecuencia con que este fenómeno aparece en las páginas de los distintos periódicos nacionales, pero que, por lo general, se concentran en sucesos, tales como: agresión con arma de fuego o punzocortantes hacia algún compañero o profesor, vandalismo dentro de la institución, amenazas e intimidación, pleito entre estudiantes, entre otros títulos. Pero también existen expresiones de violencia que pasan a veces desapercibidas, cuyas manifestaciones tienen relación con el currículo oculto y la resistencia generada por el estudiantado.

En Costa Rica, los hechos de violencia de mayor relevancia, referentes a conductas agresivas físicas en centros escolares, durante los años de este nuevo siglo, han aparecido en titulares de la prensa escrita. Esto muestra que el fenómeno de la violencia escolar es muy serio y puede ser reflejo de una sociedad violenta que no ha aprendido a solucionar los conflictos de una manera adecuada; de ahí la importancia de traer a discusión este problema, el cual se evidencia en las aulas. Asimismo, se deben buscar soluciones integrales a él. Entre estos hechos, se describen los siguientes:

- 9 de mayo del 2001, un estudiante del Liceo [...] hirió con un puñal a dos compañeros.

- $\quad$ Febrero 2003, en el Liceo [...] en Heredia, en una riña, un estudiante pierde el conocimiento y debe ser trasladado de emergencia al hospital.

- 15 de mayo del 2003, muere asesinado por un compañero un estudiante del Liceo de [...].

- 16 de febrero del 2004, dos niñas de 10 y 11 años resultaron heridas al dispararse por accidente un arma que estaba, al parecer, dentro de un bulto de un compañero, en la Unidad Pedagógica de [...], San José.

- 30 de agosto del 2005, el director del Liceo [...], fue agredido por unos estudiantes.

Esta problemática que se vive diariamente en las instituciones educativas del país exige un ejercicio de reflexión, y, de esta forma, generar discusión para hacer conciencia sobre una realidad que no puede pasar inadvertida. En este sentido, se debe investigar el problema, detectar las causas, los efectos y las posibles soluciones. Como vemos: “[...] la violencia entre escolares es un fenómeno muy complejo que crece en el contexto de la convivencia social [...] (Ortega, 1997, p. 31).

Se considera que el fenómeno de violencia escolar es aquel que provoca algún tipo de maltrato en las personas, y que se enmarca en un contexto social que ha legitimado una cultura de violencia entre iguales. Este maltrato constante y cotidiano alude a una relación estable, permanente y continua, que un joven o grupo de jóvenes establece con otros, basada en la dependencia o el miedo. Es importante entender que no se trata de fenómenos de indisciplina aislados, sino de violencia escolar, enmarcados en el hostigamiento, la amenaza, la intimidación, el robo, la agresión física o psicológica, lo cual puede ser temporal o permanente. En este sentido, muchos de los patrones de conducta agresivos son aprendidos en el medio en donde se desarrolla el joven, se inicia en el hogar, dentro de la educación informal, así que 
[...] gran parte del aprendizaje social se produce por la observación fortuita o dirigida de otras personas en las situaciones cotidianas. En los niños pequeños, el aprendizaje depende, en gran medida, del modelado de la conducta que se produce en su vida diaria. De hecho, en muchas lenguas la palabra "enseñar" significa también "mostrar", en la vida cotidiana los niños van asumiendo o incorporando conductas que les "muestran" sus padres, docentes y compañeros (Candas \& Lucero, p. 14).

\section{LA CULTURA ESCOLAR}

Los espacios sociales que se desarrollan en las aulas cobran importancia para el docente en la medida que le permiten comprender su quehacer educativo. El aula no sólo es aquel espacio físico formado por cuatro paredes, sino también el lugar en donde se desarrolla un sistema complejo de relaciones culturales, con un espacio y un tiempo específicos. Lo que ocurre en un aula responde al tiempo histórico, marcado por el ritmo de los acontecimientos sociales, económicos y políticos actuales, pero, a la vez, adquiere un sentido propio, una identidad que refleja las contradicciones propias de un sistema que se construye cotidianamente.

El aula constituye uno de los lugares más apropiados para reconocer la forma en que el estudiantado interactúa en su convivencia diaria, pues en ella, tanto educandos, como educadores comparten no solamente conocimientos, sino todo tipo de interacciones sociales, tales como valores, actitudes y comportamientos culturales. Por consiguiente, el aula es aquel espacio social y cultural organizado, intencionalmente, para el aprendizaje. En él se desarrolla un currículo oculto, caracterizado por sentidos visibles e invisibles en cuanto a las relaciones de poder, la pluralidad de saberes, los valores emergentes, los elementos de resistencias, la violencia escolar y demás interacciones horizontales ${ }^{2}$ que se expresan cotidianamente en la clase.

\section{UNA APROXIMACIÓN AL CONCEPTO DE VIOLENCIA ESCOLAR}

El fenómeno de la violencia escolar es parte de un proceso sociocultural que se construye a diario en las instituciones educativas, como parte de las interacciones sociales que en ellas se desarrollan. Por consiguiente, su estudio no es fácil, porque depende de las definidas particularidades y del contexto en que éstas suceden. Para la Organización Panamericana de la Salud (2004), la violencia es considerada como

[...] el uso deliberado de la fuerza física o el poder, ya sea en grado de amenaza o efectivo, contra uno mismo, otra persona, grupo o comunidad, que cause o tenga muchas probabilidades de producir lesiones, muerte, daños sicológicos, trastornos del desarrollo o privaciones. Esta definición considera las numerosas consecuencias del comportamiento violento, el suicida, los conflictos armados, la muerte, las lesiones, la violencia intrafamiliar. Cubre también una amplia gama de actos que van más allá del acto físico, para incluir amenazas e intimidaciones. También los daños síquicos, las privaciones y deficiencias del desarrollo que comprometan el bienestar de los individuos, las familias y las comunidades [...] (p. 6)

\footnotetext{
Representa los roles de los distintos protagonistas de la escena escolar en un proceso de socialización colectivo y recíproco, que permite el desarrollo y la construcción de nuevas relaciones y lazos entre pares.
} 
Desde este punto de vista, la violencia posee múltiples manifestaciones que se traducen en determinadas acciones de los individuos en daño directo o indirecto contra otras personas, las cuales van desde agresiones verbales o no verbales, comportamientos agresivos y conflictos interpersonales y/o sociales hasta la psicológica. De esta última se desprende la agresión verbal, que puede ocasionar situaciones de mayor conflicto entre estudiantes, como es el caso de los sobrenombres y el irrespeto, lo que pareciera ser una constante en las interrelaciones sociales, tanto dentro como fuera del aula.

Estas manifestaciones llevan explícito un lenguaje agresivo, de intimidación, de amenaza, de rivalidades y de vocabulario soez, el cual es aparece escrito en diversos lugares del entorno en que se desenvuelven los implicados, a saber: baños, pasillos, paredes, aulas, pupitres y demás espacios propicios para enviarse mensajes entre sí. Esto ocasiona rivalidades individuales y de grupo que desencadenan en la violencia física.

La violencia social, que vive la sociedad costarricense, traspasa los muros o mallas de las instituciones educativas por medio de expresiones violentas, manifestaciones disruptivas ${ }^{3} \mathrm{o}$ actitudes $^{-}$ agresivas. Los jóvenes reproducen, en las aulas, aquellos patrones violentos que han sido aprendidos en el lugar o comunidad en donde viven y socializan. Estos modelos aprendidos en la familia o en el entorno social influyen en la vida de algunos de ellos, pues son parte de un círculo de violencia de la cual ellos mismos pueden ser víctimas de la situación. En este sentido, se plantea que

[...] el aprendizaje de conductas, la observación de un modelo es un elemento facilitador poderoso. Los patrones de conducta parecen interiorizarse a través de la identificación con un modelo. La mayoría de los psicólogos actuales definen la identificación como una imitación global, general, es decir, una tendencia a imitar una variedad de características y conductas de otra persona (Candas \& Lucero, 2000).

Podría entenderse, entonces, que la imitación es un proceso de interacción social basado en la transmisión de patrones de conducta, de un sujeto a otro, por medio de símbolos, señales y sistemas de mensajes como parte del proceso de socialización, y que se expresa mediante el nexo, la relación, la vivencia o el diálogo que se establece entre las personas. En este sentido, un joven puede identificarse con un familiar e imitarle, pero también del grupo de pares, de vecinos o de programas de televisión que transmiten valores, conductas, acciones, palabras, gestos u otro tipo de manifestación violenta, y asimilarla a la vida cotidiana, como una forma de comportamiento, socialmente aceptada.

Los comportamientos violentos se legitiman y pasan a formar parte de la cultura escolar, que día a día son fuente de información en los medios televisivos y escritos, pero con un tinte amarillista. Este tipo de información masiva, puede provocar que los patrones de conducta que observan los estudiantes sean imitados y traídos a las aulas como parte de un proceso de reproducción cultural. En este sentido, la violencia responde a un contexto más amplio originado en la sociedad costarricense, como parte de una crisis en las instituciones sociales, tales como: la educación, la salud, la vivienda y la economía, en general.

Según Arias, Feoli y Fernández (2001) para comprender la violencia hay que hacer una contextualización histórica del fenómeno, y manifiestan que ésta siempre ha existido. En consecuencia, enmarcan la violencia como "[...] aquel acto generado en contra de la voluntad de la persona, debido

Conducta inapropiada por parte de uno o varios estudiantes, los cuales obstaculizan el desarrollo de la clase y que se expresa por la falta de cooperación, insolencia, desobediencia, provocación, agresividad, entre otras. Se muestra en forma verbal, por medio de gestos e interrupción de la clase. La disrupción dificulta el aprendizaje y las relaciones interpersonales. 
a sus costumbres, educación, religión y las enseñanzas transmitidas por la cultura" (p 14). Sobre este particular, concluyen que la violencia se desenvuelve en un contexto escolar homogeneizado, autoritario y en el marco de un currículo tradicional, que no toma en cuenta las diferencias y las necesidades individuales del estudiantado, y en un ambiente de aula con patrones de comunicación verbal y de gestos agresivos.

Cascante (2002) aborda el término de violencia escolar como una forma de reproducción cultural, lo cual se manifiesta de diferentes maneras, pero caracterizado por la represión, la marginación, la discriminación y las actitudes hostiles, entre otras. De esta manera, plantea que ésta debe verse desde tres enfoques diferentes, denominados violencia directa (golpes, amenazas, actos que atentan contra la integridad física de otras personas, de objetos o lugares), estructural, (violencia contra la dignidad) y violencia cultural, la cual se refiere a patrones o conductas que se reproducen socialmente.

En consecuencia, la violencia escolar tiene un carácter multidimensional y depende del contexto social y cultural en el que el fenómeno se vive y se reproduce cotidianamente. En este sentido, Hernández, Martínez y Torres (2006) la definen como

[...] una construcción multidimensional compuesta de aspectos cognitivos, afectivos y de conducta, que se manifestarían como creencias y actitudes negativas sobre los demás, ira o cólera y acciones que pretenden dañar a los demás verbal o físicamente. Se entiende por acoso escolar o bullying ${ }^{4}$ una agresión específica caracterizada por conductas dirigidas a molestar, repetidas en el tiempo y donde hay un desequilibrio de poder, de manera que los demás agreden física o psicológicamente a los de menos poder. (p. 3)

Por lo anterior, las manifestaciones de violencia escolar implican aquellas conductas de maltrato, intimidación y agresión entre jóvenes, dentro o en los alrededores de la institución educativa y/o en los horarios o momentos inmediatamente anteriores al ingreso o posteriores al egreso de ella. Además, se caracterizan porque pueden enfocarse desde comportamientos agresivos, tanto individual como en forma colectiva; psicológica por medio de gestos, maltrato verbal, intimidación, marginación, amenazas y cultural, al reproducirse patrones de conducta en las interacciones sociales del estudiante con el medio y que son reflejo de un currículo oculto, como forma de resistencia al currículo institucional. Además, las formas de violencia tienen lugar en los espacios del centro educativo o aledaños a él, tales como los pasillos, las aulas, el comedor, los baños, el gimnasio y los alrededores de la institución.

Existen diferentes tipos o formas de violencia escolar que se dan en los centros educativos, pero no existe un criterio unificado sobre ellas. Por lo general, se acostumbra a crear estereotipos, y se cae en generalizaciones ambiguas o simplistas. Por tanto, hay que tener en cuenta que no todas las acciones consideradas violentas, entre estudiantes, pueden agruparse en una misma categoría, ya que existe violencia de tipo psicológico, de tipo físico que atenta en contra de la integridad personal o aquella que se reproduce culturalmente, entre otras.

La violencia psicológica, se manifiesta por medio de agresiones verbales, gestos, intimidación, maltrato, amenazas, marginación, u otras. Para este tipo de manifestación de violencia se ha utilizado el término inglés de bullying, el que se relaciona con los procesos de intimidación y de

Alude a una concepción de violencia escolar proveniente del mundo anglo, que traducido a nuestro idioma alude al matonaje, y más específicamente, al carácter de intimidación, hostigamiento y victimización que presentarían estas conductas entre estudiantes. 
victimización entre iguales, lo que Bernal, citando a Ortega y Mora-Merchán, (2006) vincula con procesos en los que uno o más alumnos acosan e intimidan a otros por medio de insultos, rumores, vejaciones, aislamiento social, motes, entre otras maneras.

La violencia verbal es aquella forma de expresión que trata de humillar, ofender, maltratar o denigrar a una persona y forma parte de los insultos, choteo, sobrenombres, amenazas y otras conductas que pueden manifestarse oralmente o en forma escrita. Este tipo de conducta se define como "[...] un acto de violencia psicológica que se manifiesta por medio de palabras que atacan o injurian; que llevan a creer lo falso o que hablan falsamente de una persona" (Herrera, Peraza y Porter, 2004, p. 8). Lo anterior lleva implícita una relación de poder entre pares, que da como resultado que el estudiante agredido tenga baja autoestima, se sienta aislado, con depresión, enojo, frustración, entre otras manifestaciones. Este tipo de conducta agresiva puede expresarse en forma directa o indirecta.

\section{LA RESISTENCIA Y EL CURRÍCULO OCULTO}

Durante el desarrollo de la lección, el estudiantado aprende a rebelarse y a protestar contra las normas establecidas por el docente, esto se llama resistencia. Algunos estudiantes se dedican, en algún momento, a disimular los fallos en el cumplimiento, así, por ejemplo, realizan tareas de otras materias, copian durante el desarrollo de los exámenes, se burlan del docente, tiran papeles, no ponen atención, conversan temas ajenos a la lección, molestan, llegan tarde, utilizan el teléfono celular, escuchan música, se escapan, se burlan, y otras manifestaciones de este tipo. La resistencia genera en el aula una cultura que representa un espacio social de contestación que se construye mediante el currículo oculto y se legitima durante las interacciones sociales. En este sentido, Blandón, Molina y Vergara (2005) manifiestan que

se puede evidenciar cómo se reproducen en la escuela prácticas educativas, que, muy sutilmente, generan cierta violencia y ciertas reacciones en el estudiante tendientes a repeler el tipo de modelo implementado por el maestro; la imposición crea en los estudiantes un repudio que se manifiesta en el rechazo a la norma, e, incluso, a las mismas actividades curriculares. El desconocimiento por parte del docente de sus interlocutores, hace que éstos asuman una actitud defensiva y poco positiva para lograr entornos de aprendizaje mutuos en los que haya verdadera producción de conocimiento. (p. 12)

Para Ortega (1997), el currículo oculto está formado por “[...] el conjunto de procesos que discurren por debajo del control educativo que el profesorado realiza de forma consciente y planificada" (p. 31). Por consiguiente, la práctica educativa que se desarrolla en los salones de clase evidencia comportamientos valorativos que tienen estrecha relación con los mensajes verbales o simbólicos que los estudiantes expresan durante la lección y que forman parte de una cultura de contestación que se reproduce en las interacciones sociales entre pares.

La resistencia al currículo establecido se observa en el ausentismo, los escapes, la destrucción de la planta física, el rayado de pupitres y paredes. Sobre este particular, para investigadores como Funnk y Mooij, (1997) existe una relación contrastada entre el currículo escolar, los métodos de enseñanza y el agrupamiento del alumnado con la aparición de fenómenos de comportamiento antisocial. En este sentido, se considera la disrupción en el aula como una forma de expresar resistencia por medio del currículo oculto. Cuando se habla de disrupción, se expresan aquellas situaciones en 
las que tres o cuatro alumnos impiden, con su comportamiento, el desarrollo normal de la clase, lo que obliga al profesorado a emplear cada vez más tiempo en controlar la disciplina y el orden. De esta manera, se trata de conductas que implican una mayor o menor dosis de violencia, desde la resistencia o el boicot pasivo hasta el desafío y el insulto activo al profesorado, que pueden desestabilizar por completo la vida cotidiana en el aula. Al respecto, Blandón et al. (2005) concluyen que la imposición crea en los estudiantes un repudio que se manifiesta en el rechazo a la norma, e, incluso, a las mismas actividades curriculares.

Entonces, es primordial conocer de antemano las premisas fundamentales sobre las que se construyen las relaciones en el aula. En este sentido,

[...] los estilos de enseñanza directivos, que han predominado en las prácticas de la educación [...], han reproducido todo un entramado de relaciones de poder entre el profesorado y el estudiantado, caracterizado por el disciplinamiento, la normalización, la competencia y la homogeneización, lo que ha privilegiado una lógica asimétrica en las relaciones escolares, generando marcos de violencia y reproduciendo la lógica de los conflictos que padece la sociedad en su conjunto (Blandón et al., 2005, p. 11).

De esta manera, se encasilla al estudiantado en un mismo sistema, por medio de métodos y técnicas homogenizadoras. Al respecto, Vásquez y Martínez (1996) afirman que “[...] la escuela está organizada de manera que, durante las clases, el estudiantado trabaje sobre las lecciones que propone, explica y desarrolla el maestro, de modo que no se admite que los alumnos desarrollen otras actividades" (p. 17). Un claro ejemplo que demuestra esta realidad es el manejo de un concepto de disciplina tradicional, al utilizarse mecanismos de control (boletas, sanciones o castigos) cuando los estudiantes violan las normas impuestas en el salón de clase o dentro de la institución, y rompen con el rol tradicional de la escuela y el docente. De ahí que, "[...] muchos maestros se sienten abrumados y frustrados por la tarea de mantener un ambiente de aprendizaje en el salón de clases" (Gordon, 1982, p. 28).

Esta comparación entre currículo y práctica educativa, manifiesta características del enfoque curricular técnico, que por su forma rígida al desarrollar el proceso educativo, da como resultado un incremento de las manifestaciones de resistencia entre el estudiantado. Por consiguiente, los castigos, las boletas disciplinarias, la suspensión o el rebajo de puntos en las calificaciones de conducta, no han resuelto el problema de la violencia y, muchas veces, resultan en mecanismos de exclusión educativa.

La violencia es un fenómeno social que se vivencia en las aulas costarricenses, nos rodea y la mayoría de las veces la percibimos como una presencia invisible, y no logra solucionar el problema. Por esto, es fundamental que el docente desarrolle destrezas y habilidades como investigador; que pueda descifrar significados, identificar problemas y actuar consecuentemente con lo que dice y con lo que hace, a partir de la comprensión, la vivencia y la reflexión de su experiencia diaria, y es, en este sentido, que este ensayo, pretende abordar esta problemática social.

La comprensión del fenómeno de violencia escolar puede generar en la construcción de mecanismos de prevención y contención de dicha problemática. La estrategia estaría encaminada en poner en marcha las medidas necesarias para impedir la aparición de acciones violentas en los centros educativos. Ante este panorama, la educación debe proyectarse hacia una nueva etapa de desarrollo, no sólo promoviendo cambios materiales, científicos y técnicos, sino, lo que es todavía más importante, formar valores y aspiraciones humanísticas.

En el proceso de enseñanza, los y las docentes nos olvidamos que educar es formar personas, y permitirles el desarrollo pleno de sus capacidades, no solamente cognitivas, sino, también, socio- 
afectivas, para que puedan involucrarse en la sociedad en la cual viven. En este sentido, Abarca (2001) afirma que "[...] la enseñanza, es el mejor medio para formar virtudes y constituye la verdadera riqueza de nuestra patria, pues orientada a través de la instrucción y de la formación moral, representa el factor más importante para alcanzar un efectivo desarrollo integral y un conveniente bienestar integral" (p. 42). De lo anterior, se deduce que los procesos de enseñanza y de aprendizaje deben tener como meta la formación integral del estudiantado, y que el discurso del docente debe ser consecuente con las acciones llevadas a cabo en el salón de clase.

\section{CONSIDERACIONES FINALES}

Las causas de la violencia escolar son múltiples y, por tanto, se expresa como fenómeno social y cultural, debido a que son prácticas aprendidas como parte de las interacciones sociales que realiza el estudiante con el medio en el cual vive y/o estudia. Sus manifestaciones van desde actitudes verbales tales como apodos, burlas, insultos o agresiones físicas. La agresión verbal es un tipo de agresión psicológica que puede ocasionar situaciones de mayor conflicto entre estudiantes. Estos comportamientos se reflejan en el lenguaje oral y escrito con frases ofensivas en las paredes de las aulas, en los pupitres, en las pilas, los baños y los pasillos. Además de esto, se observa en los medios televisivos manifestaciones de violencia física, por medio de pleitos entre estudiantes.

El fenómeno de la violencia escolar representa aquellas conductas de maltrato, intimidación, agresión verbal o física entre pares; ésta tiene una estrecha relación con el contexto familiar y educativo en que están inmersos los educandos. En el primer caso, los estudiantes aprenden a ser agresores desde el hogar o la comunidad en donde viven, repiten esos patrones de conducta en el centro educativo mediante las interacciones que mantienen con otros. En segundo lugar, esos comportamientos aprendidos en el hogar se reproducen en la institución educativa, como parte de un proceso de socialización.

La violencia también tiene su espacio, forma parte de una cultura escolar que tiene significado para el estudiante en la medida que comparten, con un círculo restringido de compañeros, todo tipo de interacciones. Además, el espacio social le brinda al estudiante un sentido de pertenencia y seguridad que debe ser protegido a toda costa, y que puede generar rivalidad entre niveles o grupos.

En consecuencia, la violencia social que vive la sociedad costarricense se refleja en las instituciones educativas por medio de comportamientos violentos, manifestaciones o actitudes agresivas de los y las jóvenes y su incapacidad para resolver problemas ante situaciones de conflicto. Éstos reproducen patrones violentos en las aulas que son aprendidos en el lugar o comunidad en la cual viven y socializan.

Algunas de las manifestaciones de violencia que se viven en las instituciones educativas son potenciadas por el modelo curricular vigente, lo que genera reacciones o resistencia a las normas institucionales y a los reglamentos; así como el sabotaje a las clases, en general; tal es el caso del ausentismo, los escapes, la burla, el rayar o la destruir del mobiliario del aula, lo que genera un ambiente poco propicio para el aprendizaje. La respuesta del docente ante estas situaciones aparece por medio de la intimidación o la amenaza por medio de las boletas, lo cual no soluciona el conflicto.

Por consiguiente, el currículo técnico ${ }^{5}$ genera manifestaciones ocultas que propician un clima inadecuado para el manejo de situaciones violentas y que más bien favorece que éstas se potencien.

Considera el currículo en un contexto caracterizado por la planificación y el desarrollo de programas para alcanzar ciertos objetivos, a fin de transmitir valores, conductas y conocimientos que reproducen el "status quo" social. Se evidencia por medio de un modelo pedagógico homogenizador. 
Este modelo tiende a crear elementos de resistencia en el estudiantado, tales como el desacuerdo con las actividades del docente, enojo ante las boletas o castigos, poca responsabilidad ante los trabajos que deja el profesor, mala comunicación y una interacción directiva y vertical de la clase. Por tanto, los patrones de conducta violenta son reproducidos en la institución mediante prácticas curriculares homogenizadoras, que no toma en consideración las diferencias individuales, sociales o culturales.

En la institución, adquiere especial trascendencia el trato que se establece entre docentes y estudiantes, así como el que los estudiantes mantienen entre sí. Después de la familia, que es el primer lugar en el que se vive y nos educamos, la escuela es la entidad más importante, pues es el lugar en el que recibimos la enseñanza desde los primeros años. No obstante, parece que los y las estudiantes traen sus problemas a las aulas y muchas de sus respuestas están relacionadas con alguna manifestación de violencia. Por esta razón, es necesario insistir en que se debe mejorar la comunicación dentro de las interacciones sociales que tienen lugar en las instituciones educativas.

La educación tiene como fin el desarrollo integral de la persona; por eso debe proporcionar, además de conocimientos, los valores como el respeto, la convivencia pacífica, la tolerancia, entre otros. Además, la comunicación debe formar parte central en la búsqueda de espacios alternativos para que los estudiantes logren una verdadera convivencia social, donde aprendan a respetar a Ios otros y la institución busque alternativas distintas a los castigos tradicionales, que ayuden a formar la capacidad para aceptar el error y enmendarlo. Es, por tanto, que la tarea preventiva requiere investigar el problema, detectar sus causas, sus efectos y sus posibles soluciones. Esto se puede lograr mediante la participación estudiantil y comunal, que, en conjunto con los docentes, el departamento de orientación, los padres y las madres de familia, puedan llevar a cabo acciones que posibiliten prevenir el surgimiento de actos de violencia. La prevención es poner en marcha las medidas apropiadas para impedir la aparición de acciones violentas en los y las estudiantes.

Por otra parte, se debe entender que los conflictos forman parte de las relaciones humanas, por lo que no es extraño que se presenten en los salones de clase. Por consiguiente, para Gordon "[...] es común que los maestros encuentren situaciones en las que sus mensajes ya no son eficaces para modificar los comportamientos inaceptables, o en que sus esfuerzos para modificar el ambiente del salón de clases no dan resultado" (1982, p. 25). Por esto, es necesario que el profesorado desarrolle estrategias pedagógicas para manejar con éxito esas situaciones, en las que la resolución de conflictos se vuelva una tarea exitosa y no tan acongojante en el quehacer cotidiano en las aulas: los escapes, el hacer trampa o fraude, el plagiar, la rebeldía, el vandalismo, la resistencia o los desafíos pueden encontrar un punto de escape en una buena comunicación y manejo de las interacciones sociales que se desarrollan en el ambiente escolar.

De lo descrito, se interrelacionan cuatro supuestos que pueden ser causas de la violencia escolar, a saber:

a) La violencia escolar es producto de la incapacidad del joven para resolver los problemas con sus compañeros y sus profesores en el espacio institucional, por lo que su respuesta ante el conflicto es por medio de un comportamiento agresivo, que se manifiesta en el maltrato verbal o físico.

b) Algunas de las manifestaciones de violencia son aprendidas en el hogar o la comunidad de los que proviene el estudiante, mediante un proceso de socialización, culturalmente aceptado.

c) Los medios de comunicación transmiten patrones de conducta agresivos, que los y las jóvenes imitan, y comparten por medio de las interacciones horizontales.

d) El currículo escolar, al reproducir prácticas pedagógicas directivas no deja espacio para que el joven se exprese con autonomía, lo que propicia resistencia por medio del ausentismo, la deserción y el rechazo a la norma. 
Es importante considerar que no existe una receta única para solucionar el problema de la violencia escolar, ya que cada región, comunidad, institución y personas son diferentes y presentan necesidades muy particulares. Por lo anterior, es imperioso investigar el problema, diagnosticar las causas a partir de una comunicación más eficaz y dialógica, y adaptar el currículo a las necesidades reales del estudiantado. Por consiguiente, hay que buscar otros modelos de mediación pedagógica más democrática y menos autoritaria; además de la elaboración de proyectos alternativos, en los que los y las estudiantes sean partícipes y protagonistas en la construcción de ambientes de mayor apertura y, por último, desarrollar estrategias de mediación alternativa para la resolución de conflictos personales.

\section{REFERENCIAS}

Abarca Díaz, M. (2001). Fruto de la perseverancia. Sistema nacional de comisiones de valores de Costa Rica. San José: Editorial de la Universidad de Costa Rica.

Arias, M., Feoli, D. \& Fernández, M. (2001). Interacciones violentas educador-educando en el aula y en el ámbito escolar. Proyecto para optar por el título de Maestría en Psicopedagogía de la Universidad Estatal a Distancia.

Bernal, F. (2006). La violencia en el ámbito escolar. Recuperado el 15 de abril de 2008, de www. apse.org.cr/webapse/docen/docen09.htm

Blandón, M., Molina, V. A. \& Vergara, E. (2005). Los estilos directivos y la violencia escolar: las prácticas de la educación física. Revista Iberoamericana de Educación, Nº 38, 87-103.

Candas, S. \& Lucero, C. (2000). Violencia escolar. Primer Congreso Hispano-Portugués de Psicología: hacia una psicología integradora, realizado en Santiago de Compostela, España, del 21 al 23 de setiembre de 2000.

Cascante, J. (2002). Voces que callen, mentes que hablen: Una Perspectiva de la violencia en un aula escolar. Tesis para optar al título de Maestría en el Estudio de la Violencia Social y Familiar de la Universidad Estatal a Distancia.

Funk, W. \& Mooij, T. (1997): Por la seguridad en la escuela. Revista Educación, $N^{o}$ 313, 29-52.

Gordon, T. (1982): Maestros eficaz y técnicamente preparados. México: Editorial Diana

Hernández, I., Martínez, C. \& Torres, A. (Julio-Agosto, 2006). Percepción de la violencia escolar por parte de las personas responsables de la dirección de los centros de enseñanza de Alicante: un estudio cualitativo. Revista Española de Salud Pública, Vol. 80, № 4, 387-394.

Herrera, C., Peraza, C. \& Porter, H. (Marzo, 2004). El abuso verbal dentro de la violencia doméstica. [versión electrónica], Medicina legal de Costa Rica, Vol. 21, № 1, 45-90. Disponible en http:// www.scielo.sa.cr/scielo.php?pid=S1409-00152004000100005\&script $=$ sci abstract 
Ortega, R. y colaboradores. (1997). La Convivencia Escolar: qué es y cómo abordarla. Programa Educativo de prevención de Maltrato entre compañeros y compañeras. Andalucía, España: Consejería de Educación y Ciencia.

Organización Panamericana de la Salud. (2004). La violencia social en Costa Rica. Ministerio de Salud. San José, Costa Rica: Autor.

Vásquez, A. \& Martínez, I. (1996) La socialización en la escuela. Una perspectiva etnográfica. La cara oculta de la Luna: las interacciones entre alumnos en la clase. Barcelona, España: Paidós.

Otras fuentes consultadas

Campos, E. (2004). Influencia del manejo del enfoque curricular empleado por la y el docente de español y matemática en las manifestaciones de violencia vinculadas con el género en las y los estudiantes de décimo año en un colegio público de secundaria en la zona urbana de San José. Tesis para optar por el título de Doctorado en Educación de la Universidad Estatal a Distancia.

Cubero, C. M. (2004) La disciplina en el aula: reflexiones en torno a los procesos de comunicación. Revista Electrónica Actualidades Investigativas en Educación, Vol. 4, Nº 2.

Denis, L. (1995) Investigación cualitativa sobre valores en el aula. Recuperado el 20 de abril de 2008, de www. Tripot.com/denissntana/tesis/index.htlm

Moral, M. de la V., Ovejero, A. \& Pastor, J. (2000) Aprendizaje Cooperativo. Un eficaz instrumento de trabajo en las escuelas multiculturales y Multiétnicas del siglo XXI. Revista Electrónica Iberoamericana de Psicología Social: Vol. 1, N 1, 7.

Moreno Olmedilla, J. M. (2000). Comportamiento antisocial en los centros escolares: una visión desde Europa. Página del Centro de Profesores y Recursos de Latina-Carabanchel (Madrid). Recuperado el 15 de abril de 2008, de http://centros5.pntic.mec.es/cpr.de.la.latina.carabanchel/ convivir/aport.html 